\title{
Sintomatología depresiva en embarazadas víctimas de abuso sexual
}

\author{
Cristina Haydée Arrom Suhurt ${ }^{\mathrm{I}}$, Monica Routi ${ }^{\mathrm{I}}$, Margarita Samudio ${ }^{\mathrm{I}}$, Elizabeth Orue ${ }^{\mathrm{I}}$, Carmen Marina \\ Arrom $^{\text {II }}$ \\ I. Instituto de Investigaciones en Ciencias de la Salud (IICS) de la Universidad Nacional de Asunción, \\ Paraguay \\ II. Centro para el Desarrollo de la Investigación Científica CEDIC/Díaz Gill, Paraguay
}

Cómo referenciar este artículo/ How to reference this article:
Arrom C, Ruoti M, Samudio M, Orué E, Arrom CM. Sintomatología depresiva en embarazadas víctimas de abuso sexual. Mem. Inst. Investig. Cienc. Salud. 2015;13(3):82-87

\section{RE S U M E N}

Se pretendió determinar la presencia de sintomatología depresiva en embarazadas víctimas de abuso sexual que acudieron a control pre natal en Centros de Atención de Alto Paraná. Estudio descriptivo, trasversal. Muestreo consecutivo de 1049 mujeres de cuatro hospitales públicos. Como instrumento se utilizó un cuestionario anónimo que incluyó la Escala de Tácticas de Conflicto de Gelles y Straus, completado por 20 estudiantes de la Carrera de Psicología. Además se incluyó un listado de síntomas del estudio Salud Mental y Hábitos Tóxicos en Paraguay (1991) asociados al síndrome depresivo y el documento de OPS para detección de casos en Atención Primaria en Salud. Se aplicó estadística descriptiva y analítica a un nivel de significancia de 0,05 (chi-cuadrado). Un 54,5\% (570) de la población en estudio tenía menos de 24 años; 42,6\% (447) primaria completa, incompleta y sin educación formal; $83,2 \%$ casada y conviviendo, 15,3\% (160) solteras, $1,5 \%$ (16) separadas. Un 59,6\% (625) era ama de casa y sólo 20,6\% (216) tenía actividades remunerativas. La depresión se asoció a violencia psicológica $(p<0,001)$ y a violencia física $(p<0,001)$. La actividad laboral fue factor protector contra la depresión ( $p$ $=0,0099)$. Se encontró una tendencia a la asociación $(p<0,07)$ entre inactividad laboral de la pareja y depresión. La violencia conyugal constatada adquiere relevancia por la repercusión en la salud mental, mayor probabilidad de riesgos en parto, deficitario control prenatal, abortos espontáneos, trabajo de parto y parto prematuros, traumatismos fetales, bajo peso o bebés pequeños para la edad de gestación, como lo señalan algunos estudios.

Palabras clave: embarazo, violencia, depresión, factores asociados

\section{Depression in pregnant victims of sexual abuse}

\section{A B S T R A C T}

It was intended to determine the presence of depressive symptoms in pregnant victims of sexual abuse who came to pre-natal control at Care Centers of Alto Parana. It is a descriptive, cross-sectional study with consecutive sampling of 1049 women from four public hospitals. An anonymous questionnaire that included the Conflict Tactics Scale created by Straus and Gelles was used and completed by 20 students from the School of Psychology. Besides a list of symptoms associated with depressive syndrome from the Mental Health and Toxic Habits Study in Paraguay (1991) and document of PAHO fro case detection in primary health care were included. Descriptive and analytical statistics were applied to a 0.05 significance level (chi-square). The $54.5 \%$ (570) of the study population was under $24 ; 42.6 \%$ (447) had complete, incomplete and no formal primary education; $83.2 \%$ was married or living together, $15.3 \%$ (160) was single and $1.5 \%$ (16) was separated. The $59.6 \%$ (625) was housewife and only $20.6 \%$ (216) had income-generating activities. Depression was associated with psychological violence $(p<0.001)$ and physical 
violence $(p<0.001)$. Work activity was a protective factor against depression $(p<0.0099)$. A trend to association $(p<0.07)$ between the work inactivity and depression was found. Proven domestic violence becomes relevant for its impact on mental health, more probability of risks in childbirth, prenatal control deficit, spontaneous abortions, premature labor and birth, fetal injury, underweight or small babies for gestational age, as several studies point out.

Keywords: pregnancy, violence, depression, associated factors

\section{INTRODUCCIÓN}

Para comprender y abordar la violencia contra las mujeres se requiere comprenderla como un problema de salud pública y una violación de los derechos humanos. Para las mujeres en numerosas partes del mundo, la violencia es una de las principales causas de lesiones y discapacidad y a la vez un factor de riesgo para sufrir otros problemas de salud física, mental, sexual y reproductiva (1).

La violencia tiene consecuencias a largo plazo para las mujeres que la padecen, sus hijos y la sociedad toda por los costos sociales y económicos que implica. Numerosos acuerdos internacionales incluyendo la Declaración Universal de los derechos Humanos de las Naciones Unidas y la Declaración sobre la Eliminación de la Violencia contra la Mujer han reconocido este derecho humano como fundamental de las mujeres de vivir una vida libre de violencia (2).

La violencia infligida por la pareja es una de las formas más comunes de violencia contra la mujer (3). Incluye maltrato físico, sexual o emocional y comportamientos controladores por un compañero íntimo y no es exclusiva de algún entorno socio económico, cultural o religioso. Los agresores más comunes en la violencia contra la mujer son compañeros (o ex compañeros) íntimos de sexo masculino y los hombres tienen mayor probabilidad de sufrir actos de violencia perpetrados por desconocidos o conocidos que alguien más cercano. La expresión violencia doméstica se utiliza para describir la violencia de pareja pero también abarca el maltrato infligido a niños, niñas y ancianos o a cualquier miembro de una familia $(1,3)$.

Investigaciones recientes indican que con mucha frecuencia coexisten diferentes formas de violencia, la violencia física de la pareja va acompañada de abuso sexual y generalmente de maltrato emocional. En un estudio multipaís de la OMS (1) se encontró que entre $23 \%$ y $56 \%$ de las mujeres que alguna vez habían sufrido violencia física o sexual de parte de sus parejas también habían sufrido ambas formas a la vez (1).

En análisis comparativos de encuestas de demografía y salud en América Latina y el Caribe se observó que entre un $61 \%$ a $93 \%$ de mujeres que sufrieron violencia física de pareja en los 12 meses precedentes también padecieron violencia de tipo emocional.

Hay estudios que han demostrado que durante el embarazo, las cifras son considerables. Así, en el estudio Multipaís de la OMS se encontró una prevalencia de violencia física durante el embarazo entre 1\% (Japón urbano) y $28 \%$ (Perú provincia), en tanto que otros han evidenciado prevalencias más elevadas como $32 \%$ en Egipto y $40 \%$ en Africa (1).

En Paraguay la Encuesta Nacional de Demografía y Salud Sexual y reproductiva mostró que el $1,9 \%$ de de 6.526 total de mujeres encuestadas reportó abuso sexual antes de los 15 años, 36,0 \% violencia verbal, 17,9 \% violencia física, 5,0 \% violencia sexual, $13 \%$ dos tipos de violencia, 3,9\% tres tipos de violencia (4).

En el 2010 se reportaron más de 2000 denuncias de violencia contra mujeres según el Servicio de Apoyo a la Mujer de la entonces Secretaría de la Mujer de la Presidencia (SEDAMUR, hoy Ministerio de la Mujer) (5). En el 2011 se registraron 2.424 denuncias, 500 casos de violencia física; 1074 de violencia psicológica; 700 casos de violencia económica y 85 casos de violencia sexual. En tanto que en el 2012 se redujo a 859 denuncias (6).

Las mujeres maltratadas padecen mayor depresión, ansiedad y fobias que las no maltratadas. Los registros en el estudio Multipaís (OMS) registraron mayor sufrimiento emocional, ideas suicidas e intentos de suicidios entre aquellas mujeres que alguna vez han sido víctimas de maltrato físico o sexual que entre quienes no lo fueron (1).

Centrar los estudios en violencia de pareja es una forma eficaz y mayormente sólida de construir bases para la prevención de otras formas de abuso (3).

El presente estudio se propuso indagar posibles padecimientos de depresión, violencia y 
abuso sexual en 1000 mujeres que acudieron a su control pre natal en cuatro centros asistenciales del Departamento de Alto Paraná en el 2001.

\section{MATERIALES Y METODO}

Estudio descriptivo diagnóstico con componentes analíticos de corte transverso y de carácter cuantitativo no probabilístico; el muestreo fue consecutivo.

Previo consentimiento se utilizó un instrumento estructurado, anónimo, completado en entrevistas por alumnas del último año de la Carrera de Psicología. El mismo incluyó preguntas estructuradas, semiestructuradas, precodificadas, y otras abiertas, así como la Escala de Tácticas de Conflicto de Gelles y Strauss (7). En el cuestionario se incluyó un listado de síntomas del estudio Salud Mental y Hábitos Tóxicos en Paraguay (1991) (8) con 20 preguntas asociadas al síndrome depresivo, tomando como válida la presencia del mismo a partir de 8 respuestas positivas. También se consideró el documento preparado por la OPS para la detección de casos en Atención Primaria en Salud que considera la presencia de la patología a partir de 11 o más respuestas positivas. Para el análisis estadístico se aplicó estadística descriptiva y analítica a un nivel de significancia de 0.05 (chi-cuadrado). La muestra estuvo constituida por 1049 embarazadas que acudieron a control prenatal. La participación fue voluntaria y se proporcionó material educativo al término de las mismas en temas de violencia doméstica, salud sexual y reproductiva y consumo de substancias.

\section{RESULTADOS}

Fueron incluidas en el estudio 1049 embarazadas, de las cuales el 48,4\% $(n=508)$ tenía entre 18 y 25 años de edad, el $83,2 \%$ se encontraba casada o conviviendo, el $42,6 \%$ (447) tenía primaria completa, incompleta o no tenía educación formal. La mayoría de las participantes $(59,6 \%, n=625)$ se desempeñaba como ama de casa. Respecto a las actividades laborales de las parejas, el $82,4 \%(n=864)$ tenía actividad económica (Tabla 1).

Tabla 1. Características de las encuestadas. $n=1049$

\begin{tabular}{lll}
\hline Características & $\mathbf{n}$ & $\mathbf{\%}$ \\
\hline Edad (años) & & \\
$\quad<18$ & 120 & 11,4 \\
18 a 25 & 508 & 48,4 \\
26 a 35 & 315 & 30 \\
> 35 & 106 & 10,2 \\
Estado civil & & \\
Conviviendo & 476 & 45,4 \\
Casada & 397 & 37,8 \\
Soltera & 160 & 15,3 \\
Separada & 16 & 1,5 \\
Nivel educativo & & \\
Sin educación formal & 21 & 2 \\
Primaria incompleta & 295 & 28,1 \\
Primaria completa & 131 & 12,5 \\
Secundaria incompleta & 360 & 34,3 \\
Secundaria completa & 133 & 12,7 \\
Técnico & 5 & 0,5 \\
Universitaria incompleta & 30 & 2,9 \\
Universitaria completa & 14 & 1,3 \\
Sin información & 60 & 5,7 \\
Ocupación & & \\
Ama de casa & 625 & 59.6 \\
Realiza actividades remunerativas & 216 & 20.6 \\
Se hallan actualmente desempleadas & 172 & 16.4 \\
Es estudiante & 17 & 1.6 \\
Ocupación de su pareja & & \\
Actividad remunerativa & 823 & 78,4 \\
Desempleado & 89 & 8,5 \\
Agricultor & 41 & 4 \\
Otras (trabaja en la casa, discapacitado, jubilado, estudiante) & 7 & 0,6 \\
Desconocida & 89 & 8,5 \\
\hline
\end{tabular}


El $80,7 \%(n=847)$ de las embarazadas sufrió violencia psicológica; $20 \% \quad(n=205)$ violencia física alguna vez en la vida de pareja; 7,4\% $(n=78)$ abuso sexual por parte de sus parejas en los últimos 12 meses (Figura 1).

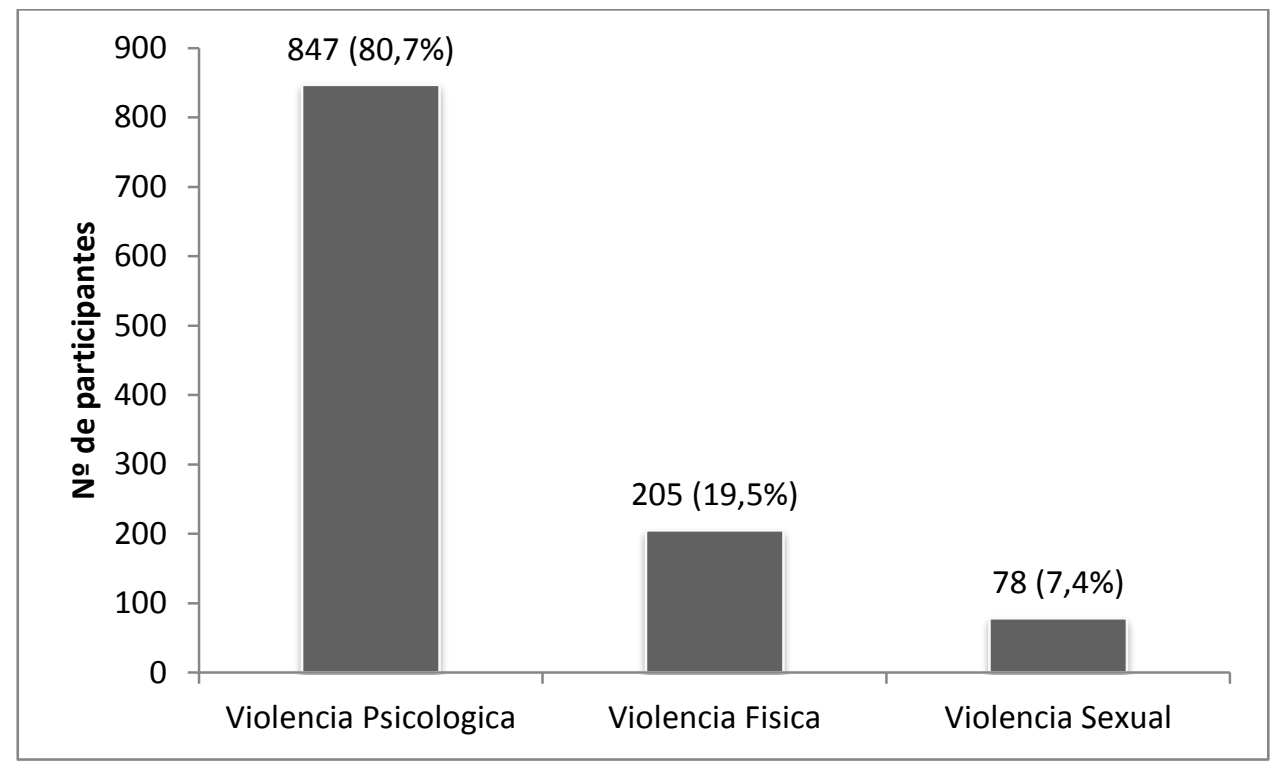

Figura 1. Tipo de violencia en embarazadas. $n=1049$

La depresión estuvo asociada a la violencia psicológica $(p<0,001)$ y a la violencia física $(p<0,001)$. Por otra parte la actividad laboral de las embarazadas fue un factor protector contra la depresión $(p=0,0099)$. Se encontró una tendencia a la asociación $(p=0,07)$ entre la falta de actividad laboral de la pareja y la depresión en las embarazadas (Tabla 2).

Tabla 2. Factores asociados a la depresión en las embarazadas.

\begin{tabular}{llll}
\hline & Con depresión & Sin depresión & Valor p \\
\hline Violencia física & $90 / 201(44,8 \%)$ & $213 / 823(25,9 \%)$ & $<0,001$ \\
Violencia psicológica & $235 / 733(32,1 \%)$ & $53 / 267(19,9 \%)$ & $<0,001$ \\
Actividad laboral & $48 / 254(18,9 \%)$ & $168 / 560(30 \%)$ & 0,0099 \\
Actividad laboral de la pareja & $49 / 236(20,8 \%)$ & $87 / 595(14,6 \%)$ & 0,07 \\
\hline
\end{tabular}

\section{DISCUSIón}

La prevalencia de violencia física encontrada coincide con el rango de prevalencia sobre violencia sufrida por mujeres durante su embarazo como el de Multipaís, principalmente el estudio en Perú provincia (1) aunque fue inferior. La violencia psicológica y combinación de más de una forma de violencia es coincidente con los estudios comparativos de las encuestas de demografía y salud realizadas en 12 países de América Latina y el Caribe donde se encontró que la mayoría de las mujeres ( $61 \%$ a $93 \%)$ que había sufrido violencia física en los 12 meses precedentes había sufrido violencia de tipo emocional. $(9,10)$, además de estudios realizados a niel país $(11,12)$.

Existe un cúmulo importante de conocimientos que se van sumando respecto a la magnitud, a las formas que adquiere, a los factores asociados a la violencia de pareja y sus efectos en la salud de las mujeres embarazadas y a la salud de los niños/as posibles testigos. Además, la mayor probabilidad de sufrir depresión, abortos espontáneos, ingresos tardíos a los servicios de atención prenatal, trabajo de parto y parto prematuros, traumatismos fetales y bajo peso al nacer o bebés pequeños para la edad de gestación, 
que constituyen factores asociados a la violencia de pareja durante el embarazo $(10,11$, 13). Pero existen aún lagunas en la investigación, por ejemplo sobre las características de las respuestas de las mujeres a la violencia y sus efectos en los niños (10).

Si comparamos los resultados con la encuesta nacional de demografía y salud, la violencia física y el abuso sexual presentan cifras ligeramente superiores (2\%) Estas diferencias pueden deberse a que el presente estudio se realizó con una población de mujeres embarazadas y no en población general, siendo la prevalencia coincidente en estudios internacionales (1) y otros estudios en el país $(11,12)$.

La sintomatología depresiva encontrada comparada a la Tasa Nacional (8) que fue en población general es superior en un $8 \%$., lo que se considera esperable en una población de mujeres embarazadas de un Servicio con un muestreo de tipo consecutivo, tal como se presentan las cifras de prevalencia en la bibliografía internacional $(1,10)$.

Esta asociación significativa entre haber sido víctima de violencia psicológica y violencia física y padecer trastornos del estado de ánimo refuerza las evidencias científicas que existentes $(1,10)$. Un estudio realizado en la Cátedra de Psiquiatría de la Universidad Nacional de Asunción encontró que los trastornos del estado de ánimo se encontraron en personas adultas que habían estado expuestas a alguna de estas formas de violencia, que se da mayoritariamente de manera combinada $(10,11,13,14)$ tanto en población infanto juvenil como adulta (15). Aún queda pendiente profundizar en estudios sobre los efectos de la exposición a diferentes tipos de violencia por la oportunidad de, sobre bases sólidas del conocimiento, construir caminos en la prevención de la violencia y otras formas de abuso en el ámbito doméstico, ya que afecta a todos los miembros del entorno como víctimas directas o como testigos en su desarrollo futuro, y genera discapacidad mental y un alto costo social para los países.(3).

La presencia de determinantes sociales como factores protectores o de riesgo a la hora de ejercer violencia hacia las mujeres se constata en la asociación significativa que muestra el presente estudio de que la actividad laboral de las mujeres constituye un elemento protector para sufrir depresión y la actividad laboral de la pareja (aunque no se mostró esto de manera significativa), como señalan otros estudios sobre el rol de la pobreza y las dificultades económicas que terminan siendo elementos que, de estar presentes, aumentan los riesgos de que mujeres sean maltratadas en el hogar $(1,10) 0$ mujeres en condiciones de vulnerabilidad $(11-13,16)$.

Las investigaciones en violencia doméstica sugieren que la influencia de la misma puede persistir mucho tiempo después de que haya cesado $(1,10)$ de manera que la vinculación o asociación entre distintas formas de violencia y sus consecuencias en la salud mental requieren persistir en los estudios. A esto se suma la complejidad de que simultáneamente se requiere atender en la práctica clínica cotidiana las necesidades inmediatas de las víctimas sin olvidar investigar sobre aquellos aspectos que han funcionado de las intervenciones para reducir el nivel general de la violencia a mediano y largo plazo. (3). En países donde predominan normas patriarcales y de supremacía de lo masculino sobre lo femenino, se legitima socialmente la violencia infligida por la pareja, lo cual se refleja en las cifras de estudios en Paraguay. Aún persiste en la sociedad paraguaya la creencia en la superioridad del hombre, el dominio que deben ejercer hacia sus parejas y los roles tradicionales de género que subordinan y ejercen sumisión sobre las mujeres. El riesgo de todo ello es que puede ser considerado normal y esperado, lo que representa un alto costo para las cifras de violencia en Paraguay $(17,18)$.

\section{REFERENCIAS BIBLIOGRAFICAS} 1. Organización Mundial de la Salud. Salud
sexual y reproductiva. Comprender y abordar la violencia contra las mujeres. /Internet/. Ginebra-Suiza: OMS; /citado 6 de abril de 2014/. Disponible en: http://www.who.int/reproductivehealth/topic s/violence/vaw_series/es/
2. Amnistía Internacional. Respeta mis derechos, Respeta mi dignidad. Módulo 3 Los Derechos Sexuales y Reproductivos son Derechos Humanos. /Internet/. London: Amnesty International Ltd; Índice: $A C T$ 30/0010/2015 Enero de 2015. /citado 6 de abril de 2014/. Disponible en: https://www.amnesty.org/download/.../ACT3 000102015SPANISH.PDF 
3. Heise LL. What Works to Prevent Partner Violence. An Evidence Overview./Internet/. Bangkok, Thailand: Centre for Gender Violence and Health. London School of Hygiene and Tropical Medicine. 2012. /citado 2 de mayo de 2014/. Disponible en: http://www2.unwomen.org/ /media/headqu arters/attachments/sections/csw/57/egm/eg m-paper-lori-

heisse $\% 20$ pdf.pdf? $v=1 \& d=20140917 T 10070$ 2

4. ONU MUJERES-Centro Paraguayo de Estudios de Población. Nuevos aportes al estudio de la violencia contra las mujeres en Paraguay, Análisis de la Encuesta Nacional de Demografía y Salud Sexual y Reproductiva 2008./Internet/. Asunción-Paraguay: ONU MUJERES-CEPEP. Octubre 2012. /citado 20 mayo 2014/. Disponible en: http://www.cepep.org.py/archivos/NUEVOS_ APORTES_ESTUDIO_VIOLENCIA_CONTRA_LA MUJER.pdf

5. ABC digital. Cada día seis mujeres sufren violencia en Paraguay. /Internet/. AsunciónParaguay: Secretaría de la Mujer de la Presidencia de la República; 07 de Enero de 2011 /citado 7 mayo 2014/. Disponible en: http://www.abc.com.py/nacionales/cada-diaseis-mujeres-sufren-violencia-en-paraguay205205.html

6. HOY. Impunidad y leyes débiles favorecen violencia contra la mujer en Paraguay. /Internet/. Asunción-Paraguay: HOY; 24 de noviembre de 2012. /citado 20 noviembre 2014/. Disponible en: http://www.hoy.com.py/nacionales/impunida d-y-leyes-debiles-favorecen-violencia-contrala-mujer

7. Mora Ríos J, Natera Rey G, Tiburcio Sainz M, Juárez F. Propiedades Psicométricas de la Escala de Tácticas de Conflicto (CTS2) en Mujeres Mexicanas. Revista Mexicana de sicología. 2008;25(1):107-17.

8. Míguez H, Pecci M, Carrizosa A. Estudio sobre salud mental y hábitos tóxicos en el Paraguay. Proyecto Marandú del Comité Paraguay Kansas. Ministerio de Salud Pública y Bienestar Social. Asunción-Paraguay: Comité Paraguay Kansas; 1991.

9. Florida International University, Latin American and Caribbean Center. Violence against women in Latin America and Caribbean. Hemisphere. 2013;22(1):4-48.
10. Organización Panamericana de la Salud. Comprender y abordar la violencia contra las mujeres. Violencia infligida por la pareja. /Internet/. Washington, DC-EEUU: OPS; 2013./citado 11 de Junio 2014/. Disponible en PDF en: http://apps.who.int/iris/bitstream/10665/988 16/1/WHO_RHR_12.36_spa.pdf?ua =1

11. Arrom C, Samudio M, Arrom CM, Lampert N, Arrom MA, Orué E. Violencia intrafamiliar, de pareja íntima y abuso sexual en puerperio inmediato. Su relación con depresión. Revista Paraguaya de Psiquiatría. 2013;1(2):17-23.

12. Galeano A, Orrego B, Pérez M, Ruiz V, Arrom $M A$, Ruoti $M$, et al. Violencia intrafamiliar sufrida por mujeres en un Hospital Público. Revista Paraguaya de Psiquiatría. 2015;3(1):42-9.

13. Arrom C, Ruoti $M$, Samudio M, Orué $E$. Condiciones socioeconómicas de adolescentes embarazadas con síntomas de depresión, consumo de sustancias y víctimas de violencia intrafamiliar. Revista Paraguaya de Psiquiatría. 2015;3(1):13-9.

14. Fresco MP, Arrom C, Samudio M, Arrom CM, Capurro M, Arrom MA, et al. Psicopatología en adultos con antecedentes de violencia intrafamiliar. Revista Paraguaya de Psiquiatría. 2015;3(1):8-12.

15. Arrom C, Ruoti $M$, Samudio $M$, Orué $E$. Sintomatología depresiva y su relación con la edad y el sexo en adolescentes estudiantes de colegios secundarios de Alto Paraná. Revista Paraguaya de Psiquiatría. 2015;3(1):33-7.

16. Arrom C, Ruoti M, Orué E, Arrom CM. El castigo y sus diferentes formas en la crianza de niños, niñas y adolescentes en 1049 familias de Alto Paraná. Revista Paraguaya de Psiquiatría. 2015;3(1):26-32.

17. Arrom C, Fresco MP, Arrom CM, Arce A, Arrom MA, Romero $M$, et al. Violencia intrafamiliar. Revista Paraguaya de Psiquiatría. 2015;3(1):38-41.

18. González Vera, M. Patriarcado, machismo e impunidad: causas de la cotidiana violencia de género. En: Coordinadora de Derechos Humanos del Paraguay (Codehupy). Derechos humanos en Paraguay/Yvypóra Derécho Paraguáipe. Codehupy. Asunción: Mercurio S.A.; 2012;447-63. 